\title{
PERFIL DAS MULHERES QUE REALIZARAM EXAME DE PAPANICOLAOU EM UMA UNIDADE DE SAÚDE NO INTERIOR DO PARANÁ
}

\author{
PROFILE OF WOMEN WHO PERFORMED PAPER SCREENING IN A \\ HEALTH UNIT INSIDE PARANÁ
}

\begin{abstract}
Nathalia Jung Ferreira Malta*, Giovana Garbelini de Souza, Maria Elisa Wotzasek Cestari, Keli Tomeleri Da Fonseca Pinto.
\end{abstract}

Universidade Estadual de Londrina, Londrina, PR, Brasil. *nathalia_jferreira@hotmail.com

Submetido em: 23/09/2019; Aceito em: 17/07/2020.

\section{RESUMO}

O objetivo deste estudo foi analisar o perfil das mulheres que realizaram o exame de Papanicolaou. Trata-se de um estudo transversal, retrospectivo e quantitativo. A população estudada foram mulheres atendidas em uma Unidade Básica de Saúde entre os anos de 2014 a 2016, totalizando 215 mulheres. A coleta de dados ocorreu por meio de fichas arquivadas na Unidade Básica de Saúde. A análise descritiva foi realizada com frequências absolutas e relativas. Evidenciou que a maior parte das mulheres apresentou idade entre $25-64$ anos $(80,9 \%)$, com companheiro $(61,6 \%)$, com trabalho remunerado (48\%), não fumantes $(71,2 \%)$, com índice de massa corpórea elevada $(50,4 \%)$, sem queixas ginecológicas $(66,8 \%)$, ativas sexualmente $(69,6 \%)$, sedentárias $(51,2 \%)$ e que não utilizavam métodos contraceptivos (35,2\%). Ressalta-se que o estudo possibilitou conhecer o perfil da população estudada, auxiliando no direcionamento das atividades em consonância com as recomendações da Política de Prevenção do câncer de colo de útero.

Palavras-chave: Atenção Primária à Saúde. Enfermagem. Saúde da Mulher. Teste de Papanicolau.

\section{ABSTRACT}

The aim of this study was to analyze the profile of women who underwent the Pap smear. This is a cross-sectional, retrospective and quantitative study. The studied population were women attended at a Basic Health Unit between the years 2014 to 2016, totaling 215 women. Data collection took place using files filed at the Basic Health Unit. Descriptive analysis was performed with absolute and relative frequencies. It showed that most women were aged 25-64 years $(80.9 \%)$, with a partner $(61.6 \%)$, with paid work $(48 \%)$, non-smokers $(71.2 \%)$, with a high body mass index (50.4\%), without gynecological complaints $(66.8 \%)$, sexually active $(69.6 \%)$, sedentary $(51.2 \%)$ and who did not use contraceptive methods (35.2\%). It is noteworthy that the study made it possible to know the profile of the population studied, assisting in directing activities in line with the recommendations of the cervical cancer prevention policy. 
Keywords: Nursing. Pap test. Primary Health Care. Women's Health.

\section{INTRODUÇÃO}

O câncer do colo de útero é um problema de saúde pública muito importante, sendo o terceiro tumor mais frequente na população feminina, estando atrás do câncer de mama e colorretal, e a quarta causa de morte em mulheres no Brasil (BRASIL, 2020a).

Este câncer é causado pela infecção persistente de alguns tipos do Papilomavírus Humano (HPV), estando presente em quase 100\% dos casos de câncer. Sendo que, o vírus apresenta mais de 150 genótipos diferentes, sabendo que 12 destes são considerados oncogênicos e associados às neoplasias malignas do trato genital (BRASIL, 2020a).

Os subtipos oncogênicos mais prevalentes são 16 e 18 e responsáveis por $70 \%$ dos casos de câncer de colo de útero. Esta neoplasia demora em média de 10 a 20 anos para progredir, e sua manifestação se dá por meio de transformações intraepiteliais (BRASIL, 2020a).

Cerca de $80 \%$ da população feminina mundialmente ativas sexualmente serão infectadas por algum tipo de HPV, porém, a maioria dessas infecções serão transitórias, combativas pelo sistema imunológico. Fatores de risco para este tipo de câncer estão relacionados à multiplicidade de parceiros sexuais, tabagismo, uso prolongado de contraceptivo oral (AMORIM, 2016).

Desde o ano de 2014 o Ministério da Saúde implementou no calendário vacinal a vacina tetravalente contra o HPV para meninas de 9 a 13 anos. Em 2017, ampliou-se para meninos de 11 a 14 anos, protegendo contra os tipos 6,11,16 e 18 do HPV (BRASIL, 2020a).

Entretanto, mulheres em atividade sexual anterior à disponibilidade da vacina e também as que estão fora da faixa etária de cobertura (9 a 14 anos), não tiveram oportunidade dessa proteção vacinal. Para esse grupo, as formas mais efetivas para a prevenção do câncer do colo de útero continuam sendo comportamentos sexuais seguros e o exame de citologia cervical, conhecido como Papanicolaou (BRASIL, 2017).

A citologia cervical deve ser realizada em mulheres a partir de 25 anos que iniciariam a atividade sexual, e com periodicidade a cada três anos, se os dois primeiros exames anuais apresentarem resultados normais, sendo 0 rastreamento preconizado até os 64 anos de idade (BRASIL, 2020a).

Com a criação do Programa Saúde da Família (PSF) em 1994, intitulado Estratégia Saúde da Família (ESF) desde 1996, aumentou-se a oferta do exame Papanicolaou em todo território brasileiro, diminuindo a incidência desse tipo de câncer ao longo das últimas três décadas, porém, as taxas brasileiras demonstram valores intermediários em relação a países em desenvolvimento (BRASIL, 2020b).

Os valores de sobrevida para este tipo de câncer em todo território mundial obtiveram melhoras de $50 \%$ para mais de $70 \%$. A última estimativa mundial realizada em 2012, demonstrou que 265 mil mulheres foram a óbito decorrente desta neoplasia, sendo que $87 \%$ destas aconteceram em países em desenvolvimento. No Brasil em 2017, ocorreram 6.385 mortes de mulheres por 
este câncer. Para o ano de 2020 espera-se uma taxa de 16.590 mil casos novos de câncer de colo uterino (AGUILAR; SOARES, 2015; BRASIL, 2020b).

$\mathrm{Na}$ Atenção Básica, as Unidades Básicas de Saúde são consideradas portas de entrada do usuário no sistema de saúde, espaço onde o enfermeiro é o profissional eficaz em uma equipe multiprofissional, para realizar a prevenção do câncer de colo uterino, geralmente, é este profissional que proporciona informações, que acolhe e demonstra à mulher privacidade durante a consulta de enfermagem, com isso, garante a partir da utilização científica, a identificação de sinais e sintomas da doença, fornecendo ações para prevenção, promoção, recuperação e reabilitação da saúde da mulher (BRASIL, 2015; SOUZA; COSTA, 2015).

Alguns fatores influenciam na adesão das mulheres ao exame de prevenção, assim é importantíssimo identificar o perfil dessas mulheres para auxiliar no planejamento das ações, com o objetivo de contribuir para o aumento dessa adesão. Ressalta-se que conhecer a população, permite ao profissional que trabalha com essa população obter informações que o auxiliará no estabelecimento de vínculo, contribuindo para o entendimento das necessidades das mulheres (BISCESK et al., 2012).

Nesse sentido, o objetivo desse estudo foi analisar o perfil das mulheres que realizaram exame de Papanicolaou.

\section{MATERIAL E MÉTODOS}

Trata-se de um estudo transversal e retrospectivo com análise quantitativa dos dados. O estudo foi realizado em uma Unidade Básica de Saúde no município de Cambé-PR, situado no norte do Paraná, região localizada no sul do Brasil (BRASIL, 2009).

O município possui uma população de pouco mais de 100 mil habitantes, tendo uma estimativa da população em 2015 de 103.822 mil pessoas, sendo 47.299 do sexo masculino e 49.434 do sexo feminino. Dessas 31.395 são mulheres em idade fértil com idade entre 10 e 49 anos e 28.037 são mulheres em idade 25-64 anos, sendo a população definida como alvo de estratégia de rastreamento do câncer de colo do útero. A região da UBS estudada tem cerca 6.541 usuários, constituído por 3.354 mulheres, destas 1.909 apresentam idade entre 25 a 64 anos, 602 com idade de 50 a 69 anos e 2.137 entre 10 a 49 anos (BRASIL, 2009).

As regiões Sul e Sudeste apresentaram as menores taxas para o câncer de útero (4,82/100.000 e 3,64/100.000 mulheres), representando a quinta e sexta posições, respectivamente, entre os óbitos por câncer em mulheres (BRASIL, 2020c).

A população do estudo foi composta por mulheres atendidas na UBS entre os anos de 2014 a 2016, que compareceram para a realização de prevenção dos cânceres ginecológicos (câncer de mamas e colo de útero), totalizando 215 mulheres.

A coleta de dados ocorreu no período de maio de 2018, através de consultas aos prontuários das mulheres atendidas. Foram analisadas as variáveis sociodemográficas e ginecológica/obstétricas: idade, escolaridade, situação conjugal, ocupação, Índice de massa corpórea (IMC), etilista, tabagista, 
número de gestações, queixas ginecológicas, ciclo menstruais, atividade sexual, atividade física e métodos contraceptivos, como variáveis independentes e a realização do preventivo como variável dependente.

Os dados obtidos foram tabulados no programa Microsoft Excel for Windows $₫ 2010$ e importados para o software Statistical Package for the Social Science, versão 20.0. A análise descritiva foi realizada por meio do cálculo de frequências absolutas e relativas.

O projeto de pesquisa foi aprovado pelo Comitê de Ética e Pesquisa da Universidade Estadual de Londrina, sob o certificado para apreciação ética: CAAE 65211816.3.0000.523.

\section{RESULTADOS E DISCUSSÃO}

A caracterização sociodemográfica das mulheres (Tabela 1) demonstrou que $174(80,9 \%)$ se encontrava na faixa etária entre 25 a 64 anos. Cerca de 85 $(34,0 \%)$ mulheres apresentaram ensino fundamental incompleto ou nenhum estudo, com relação à situação conjugal $154(61,6 \%)$ eram casadas ou estavam união estável. Relacionado a ocupação 120 (48,0\%) mulheres tinham algum tipo de ocupação, $126(50,4 \%)$ estavam com sobrepeso ou obesas. Com relação ao uso de bebida alcoólica 173 (69,2\%) referiam não fazer uso e 178 (71,2\%) não fazem uso de tabaco.

A história ginecológica e obstétrica (Tabela 2) demonstrou que 99 (46,0\%) tinha entre uma a duas gestações. Com relação as queixas menstruais 167 $(66,8 \%)$ não referiam nenhuma queixa, relacionado ao ciclo menstrual 86 $(34,4 \%)$ tinham o ciclo menstrual regular, $174(69,6 \%)$ com vida sexual ativa e $128(51,1 \%)$ praticavam algum tipo de atividade física. Relacionado aos métodos contraceptivos $88(35,2 \%)$ mulheres referiram não utilizar nenhum método contraceptivo.

A maioria das mulheres do estudo apresentou a idade preconizada (2564 anos) pelo Ministério da Saúde como ideal para a prevenção do câncer de útero, devido ao fato dessa faixa etária apresentar maior taxa de incidência de presença de lesões de alto grau no colo uterino, que se tratadas efetivamente não evoluem para câncer (ALDO et al., 2017). Um estudo realizado em Francisco Beltrão-PR, encontrou baixa adesão ao exame preventivo em mulheres acima de 60 anos (GEREMIA et al., 2016).

Segundo INCA o câncer do colo do útero é raro em mulheres até 30 anos e sua incidência maior se dá na faixa etária de 45 a 50 anos, sendo que a mortalidade aumenta progressivamente a partir de 40 anos, diminuindo após os 65 anos, pois os riscos de desenvolvimento são reduzidos devido sua evolução ser de forma lenta (INCA, 2017). Um estudo realizado em uma UBS, localizada no município de Cerro Azul, na região metropolitana de Curitiba, encontrou que as mulheres da faixa etária de 25 a 30 anos eram as que mais apresentam cobertura de exame de Papanicolaou, por procurar mais os serviços de saúde para planejamento familiar ou pré-natal (AMORIM, 2016).

No presente estudo, mulheres na faixa etária de 15 a 24 anos tiveram uma adesão baixa $(14,4 \%)$ à prevenção do câncer de colo de útero, considerado como fator preocupante, pois esta é a faixa etária mais susceptível a apresentar o HPV, lesões de baixo grau, que regredirão espontaneamente na maioria dos 
casos, devido ao fator imunológico, como também mais susceptíveis a outras Infecções Sexualmente Transmissíveis (IST's).

Tabela 1 - Caracterização sociodemográfica das mulheres que realizaram o exame de Papanicolaou em uma Unidade Básica de Saúde do Norte do Paraná

\begin{tabular}{|c|c|c|}
\hline Variável & $\mathbf{N}$ & $\%$ \\
\hline \multicolumn{3}{|l|}{ Idade } \\
\hline 15- 24 anos & 31 & 14,4 \\
\hline 25- 64 anos & 174 & 80,9 \\
\hline 65 anos ou mais & 10 & 4,7 \\
\hline \multicolumn{3}{|l|}{ Escolaridade } \\
\hline Não estudou/ ensino fundamental incompleto & 85 & 34,0 \\
\hline Ensino fundamental completo & 46 & 18,4 \\
\hline Ensino médio/graduação & 81 & 32,4 \\
\hline Sem registro & 3 & 1,2 \\
\hline \multicolumn{3}{|l|}{ Situação Conjugal } \\
\hline Casada/união estável & 154 & 61,6 \\
\hline Viúva/separada & 27 & 10,8 \\
\hline Solteira & 33 & 13,2 \\
\hline Sem registro & 1 & 0,4 \\
\hline \multicolumn{3}{|l|}{ Ocupação } \\
\hline Sem ocupação & 92 & 36,8 \\
\hline Com ocupação & 120 & 48,0 \\
\hline Sem registro & 3 & 1,2 \\
\hline \multicolumn{3}{|l|}{ IMC } \\
\hline Sobrepeso/obeso & 126 & 50,4 \\
\hline Baixo peso & 11 & 4,4 \\
\hline Adequado & 65 & 26,0 \\
\hline Sem registro & 13 & 5,2 \\
\hline \multicolumn{3}{|l|}{ Etilista } \\
\hline Sim & 38 & 15,2 \\
\hline Não & 173 & 69,2 \\
\hline Sem registro & 4 & 1,6 \\
\hline \multicolumn{3}{|l|}{ Tabagista } \\
\hline Sim & 35 & 14,0 \\
\hline Não & 178 & 71,2 \\
\hline Sem registro & 2 & 0,8 \\
\hline
\end{tabular}

Fonte: os autores.

Ressalta-se que o conhecimento deficiente, à imaturidade, o desinteresse sobre práticas preventivas, a não utilização ou a utilização inadequada de preservativos são desencadeadores para o surgimento de infecções sexuais transmissíveis (LEITE; SABRINI; BEAZUSSI, 2019). 
Tabela 2 - Caracterização da história ginecológica e obstétrica das mulheres que realizaram o exame de Papanicolaou em uma Unidade Básica de Saúde do Norte do Paraná

\begin{tabular}{lcc}
\hline Variável & $\mathbf{N}$ & $\%$ \\
\hline Número de gestação & 29 & 13,5 \\
Nuligestação & 99 & 46,0 \\
Uma a duas gestações & 87 & 40,5 \\
Três ou mais gestações & 30 & 12,0 \\
\hline Queixas ginecológica & 167 & 66,8 \\
Cólica e mal-estar & 18 & 7,2 \\
Sem queixas & & \\
Sem registro & 52 & 20,8 \\
\hline Ciclo menstrual & 57 & 22,8 \\
Amenorréia & 86 & 34,4 \\
Irregular & 20 & 8,0 \\
Regular & & \\
Sem registro & 174 & 69,6 \\
\hline Atividade sexual & 38 & 15,2 \\
Sim & 3 & 1,2 \\
Não & & \\
Sem registro & 85 & 34,0 \\
\hline Atividade Física & 128 & 51,2 \\
Sim & 2 & 0,8 \\
Não & & \\
Sem registro & 25 & 10,0 \\
\hline Tipo de método contraceptivo & 54 & 21,6 \\
Método de barreira & 88 & 35,2 \\
Método hormonal & 12 & 4,8 \\
Não utiliza método & 28 & 11,2 \\
Outros métodos & 8 & 3,2 \\
Método cirúrgico & &
\end{tabular}

Fonte: os autores.

Um estudo ocorrido em São Pedro-PI propôs uma parceria entre os profissionais da educação e da saúde, para que juntos pudessem alcançar um maior número desta população e melhorar o nível de conhecimentos destes sobre a prática segura do sexo.

A escolaridade da maioria das mulheres encontrada nesse estudo foi menor que 8 anos de estudo, podendo ser considerada baixa.

Outros estudos apresentaram diferentes realidades, em que a metade da população apresenta ensino médio completo, ou seja, 11 anos de estudo (NICOLAU et al., 2015; TANAKA; MATSUZAKA; SASAKI, 2018). Ressalta-se que a população de baixo nível de escolaridade está mais susceptível a adoecer e apresenta maior propensão de não realizar os exames preventivos. A baixa escolaridade prejudica a compreensão da importância do exame de Papanicolaou (NICOLAU et al., 2015; TANAKA; MATSUZAKA; SASAKI, 2018). 
Entretanto, o contrário também foi apontado por dois estudos, em que as mulheres mais escolarizadas e com maior renda familiar, foram as que menos obtiveram comparecimento ao exame (GEREMIA et al., 2016; GIANINO et al., 2018).

Com relação à situação conjugal, os dados encontrados foram convergentes a outro estudo que encontrou que a maioria das mulheres que compareciam ao exame eram mulheres com companheiros (BARCELOS et al., 2017).

Um achado interessante, foi de que mais da metade das mulheres $(56,6 \%)$ apresentavam trabalho remunerado, e isso não dificultou a adesão ao exame. A inserção da mulher no mercado de trabalho parece prejudicar o comparecimento nas UBS para realização de exames preventivos, pois ocorre conflito de horários entre seu trabalho e o expediente das Unidades Básicas (AGUILAR; SOARES, 2015; OLIVEIRA, A. E. C. et al., 2016).

No que concerne ao número de gestações, os resultados apresentaram que a maioria das mulheres teve de um a três filhos, constituindo uma média de 2,27 filhos por mulheres, número acima da média da região sul do país, que é de 1,78 no ano de 2010 (OLIVEIRA, P. S. D. et al., 2016).

A maior parte das mulheres não realizavam atividade física $(51,2 \%)$ e estavam obesas/sobrepeso (50,4\%). Ressalta-se que a prática de atividade física regular e o peso adequado mostram-se importantes funções preventivas contra os cânceres (BRASIL, 2018).

Entretanto, demonstraram o comportamento preventivo, no que diz respeito a não ingestão de bebida alcoólica $(69,2 \%)$ e não uso de tabaco $(71,2 \%)$. Um estudo realizado no Ceará, encontrou que a maioria das mulheres fazia uso destes (NICOLAU et al., 2015). Sabe-se que o tabaco diminui células que são responsáveis pela defesa do tecido epitelial, sendo que o cigarro contém mais de 300 substâncias com potencial efeito cancerígeno. Seu elevado percentual representa uma grave situação, considerando os malefícios do tabaco não só como precursor do câncer, mas também de outros agravos para a saúde do indivíduo. A dificuldade ou ausência de programas de combate ao tabagismo, prejudica esta situação, além da dificuldade de abandono do vício, onde se relacionam vários aspectos de vida (NICOLAU et al., 2015; BRASIL, 2020c).

\section{CONCLUSÃO}

O estudo possibilitou conhecer o perfil das mulheres que realizaram o exame de Papanicolaou, podendo constatar que a maioria das mulheres tinham entre 25-64 anos, tinham companheiro, exerciam trabalho remunerado, apresentavam índice de massa corpórea elevada, sendo também sedentárias, não apresentavam queixas ginecológicas, eram ativas sexualmente.

Entretanto, destaca-se que o perfil estudado dessas mulheres, evidenciou que os fatores de risco estudados (uso de anticoncepção hormonal e tabagismo) não foi evidente nesse estudo. Em contrapartida, o sedentarismo e o peso inadequado das mulheres demonstraram-se como um comportamento não preventivo para o câncer. 
Como limitação do estudo, destaca-se o fato de não ter a informação de outros fatores de risco para o câncer de útero, como a iniciação sexual precoce, a multiplicidade de parceiros.

Conhecer e analisar a população com a qual se trabalha é essencial para o planejamento de estratégias para a prevenção do câncer de colo de útero, pois permite conhecer os fatores de risco da população. Assim, esses resultados são de suma importância para direcionar as atividades da UBS em consonância com as recomendações da Política de Prevenção do câncer de colo de útero.

\section{REFERÊNCIAS}

AMORIM, C. G. HPV e câncer de colo do útero. Revista Cientifica da FASETE, 2016.

ALDO, R. F. F. et al. Estudo sobre a incidência de casos de adenocarcinoma de colo uterino no Distrito Federal. Health and Environment World Congress, v. 17, p. 86-88, 2017.

AGUILAR, R. P.; SOARES, D. A. Barreiras à realização do exame Papanicolau: perspectivas de usuárias e profissionais da Estratégia de Saúde da Família da cidade de Vitória da Conquista-BA. Physis. Revista de Saúde Coletiva, v. 25, n. 2, p. 359-379, 2015.

BARCELOS, M. R. B. et al. Qualidade do rastreamento do câncer cervical. Revista Saúde Pública, v. 51, n. 67, p. 1-13, 2017.

BISCESKI, A. et al. Características epidemiológicas da saúde materno-infantil. Revista de Enfermagem, v. 8, n. 8, p. 79-88, 2012.

BRASIL. Ministério da Saúde. Datasus. Média de anos de estudo das pessoas de 10 anos ou mais de idade por sexo. 2009.

BRASIL. Ministério da Saúde. Datasus. Estimativa população residente no município de Cambé-PR. 2015.

BRASIL. Ministério da Saúde. Secretaria Vigilância em Saúde. Departamento de Vigilância das doenças transmissíveis. Informe técnico sobre a vacina contra o papilomavírus humano (HPV). Brasília, 2017.

BRASIL. Ministério da Saúde. Instituto Nacional de Câncer José Alencar Gomes da Silva. Causas e prevenção, atividade física. 2018.

BRASIL. Ministério da Saúde. Instituto Nacional de Câncer José Alencar Gomes da Silva. Atlas de Mortalidade. 2020a. 
BRASIL. Ministério da Saúde. Instituto Nacional de Câncer José Alencar Gomes da Silva. Coordenação de Prevenção e Vigilância Estimativa 2020: Incidência de câncer no Brasil. Rio de Janeiro, 2020b.

BRASIL. Ministério da Saúde. Instituto Nacional de Câncer José Alencar Gomes da Silva. Causas e prevenção, tabagismo. 2020c.

INSTITUTO NACIONAL DE CÂNCER. Estimativa 2018: incidência de câncer no Brasil. Instituto Nacional de Câncer José Alencar Gomes da Silva. Coordenação de Prevenção e Vigilância. - Rio de Janeiro: INCA, 2017.

GEREMIA, D. S. et al. Avaliação da adequabilidade da coleta do exame citopatológico na Estratégia Saúde da Família. Revista Acreditação, v. 6, n. 11, p. 99-108, 2016.

GIANINO, M. M. et al. Organized screening programmes for breast and cervical cancer in 17 EU countries: trajectories of attendance rates. BMC Public Health, v. 18, n. 1 , p. $60-64$.

LEITE, T. H.; SALVINI, Y. F.; BEAZUSSI, K. M. Análise da percepção dos jovens da educação básica em relação a prevenção do HPV após a inclusão do processo de imunização no calendário vacinal. Revista Interdisciplinar do Pensamento Científico, v. 5, n. 5, p. 455-469, 2019.

NICOLAU, A. I. O. et al. Proximal Social Determinants Related to Cervical Cancer in Imprisioned Women. Revista Mineira de Enfermagem, v. 19, n. 3, p.725-740, 2015.

OLIVEIRA, A. E. C. et al. Adesão das mulheres ao exame citológico do colo uterino na atenção básica. Revista de Enfermagem UFPE On Line, v. 10, n. 11, p. 4003-4014, 2016.

OLIVEIRA, P. S. D. et al. Adesão das mulheres ao exame preventivo do câncer de colo de útero: um ensaio comunitário. Revista Enfermagem UFPE On Line, v. 10 , n. 2 , p. $442-448,2016$.

SOUZA, A. F.; COSTA, L. H. R. Conhecimento de Mulheres sobre HPV e Câncer do Colo do Útero após Consulta de Enfermagem. Revista Brasileira de Cancerologia, v. 61, n. 4, p. 343-350, 2015.

TANAKA, R.; MATSUZAKA, M.; SASAKI, Y. Influence of Income on Cancer Incidence and Death among Patients in Aomori, Japan. Asian Pacific Journal of Cancer Prevention, v. 19, n. 11, p. 3193-202, 2018. 